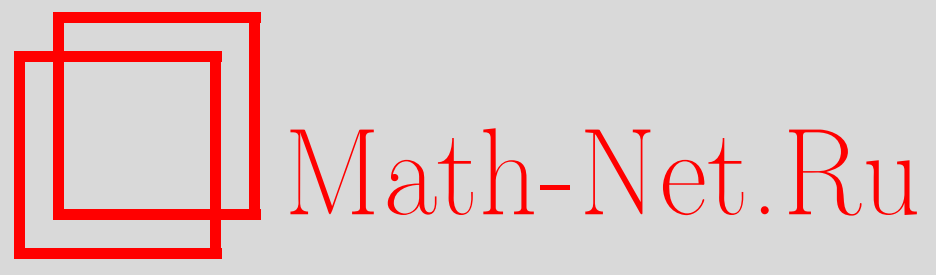

P. M. Хакимов, Локализация трансляционно-инвариантных мер Гиббса для моделей Поттса и "solid-on-solid" на дереве Кэли, $T M \Phi$, 2014, том 179, номер 1, 24-35

DOI: https://doi.org/10.4213/tmf8599

Использование Общероссийского математического портала Math-Net.Ru подразумевает, что вы прочитали и согласны с пользовательским соглашением http: //www . mathnet.ru/rus/agreement

Параметры загрузки:

IP : 54.147 .182 .235

26 апреля 2023 г., $14: 19: 47$

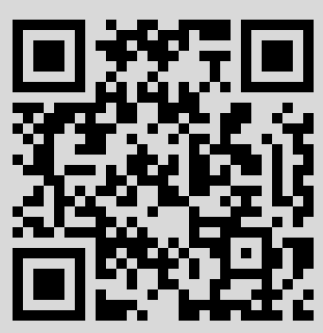




\title{
ЛОКАЛИЗАЦИЯ ТРАНСЛЯЦИОННО-ИНВАРИАНТНЫХ МЕР ГИББСА ДЛЯ МОДЕЛЕЙ ПОТТСА И "SOLID-ON-SOLID" НА ДЕРЕВЕ КЭЛИ
}

\begin{abstract}
Изучаются модели Поттса и "solid-on-solid" с $q \geqslant 2$ состояниями на дереве Кэли порядка $k \geqslant 1$. Для модели Поттса при любых значениях параметра $q$, а также для модели "solid-on-solid" при $q \leqslant 6$ найдены множества, в которых лежат все трансляционно-инвариантные меры Гиббса.
\end{abstract}

Ключевые слова: дерево Кэли, конфигурация, мера Гиббса, модель Поттса, модель "solid-on-solid", периодические меры, трансляционно-инвариантные меры.

DOI: $10.4231 / \operatorname{tmf} 8599$

\section{1. ВВЕДЕНИЕ}

Модели Поттса и "solid-on-solid" (SOS) являются обобщением модели Изинга, которая хорошо изучена на решетке $\mathbb{Z}^{d}$. Понятие меры Гиббса для таких моделей на дереве Кэли вводится обычным образом (см. монографии [1]-[4]).

Приведем обзор известных результатов. В работе [5] рассмотрена ферромагнитная модель Поттса с тремя состояниями на дереве Кэли второго порядка и дока-

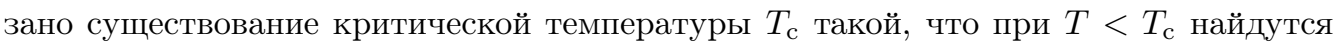
три трансляционно-инвариантные меры Гиббса и несчетное число мер Гиббса, не являющихся трансляционно-инвариантными. В работе [6] обобщены результаты работы [5] для модели Поттса с конечным числом состояний на дерево Кэли произвольного (конечного) порядка. В работе [7] доказано, что на дереве Кэли трансляционно-инвариантная мера Гиббса антиферромагнитной модели Поттса с внешним полем единственна. Работа [8] посвящена модели Поттса со счетным числом состояний и с ненулевым внешним полем на дереве Кэли. Доказано, что эта модель имеет единственную трансляционно-инвариантную меру Гиббса. Для ознакомления с другими свойствами модели Поттса на дереве Кэли см., например, монографию [4] и статьи [9]-[12]. Несмотря на многочисленность работ, посвященных модели Поттса на дереве Кэли, она изучена мало по сравнению с моделью Изинга.

*Наманганский государственный университет, Наманган, Узбекистан. E-mail: rustam-7102@rambler.ru 
В случае кубической решетки модель SOS изучалась в статье [13], где рассматривался аналог так называемой теории Динабурга-Мазеля-Синая. С другими результатами, полученными для модели SOS, можно ознакомиться в монографии [4] и статье [14].

Настоящая работа посвящена изучению трансляционно-инвариантных мер Гиббса моделей Поттса и SOS на дереве Кэли. Содержание работы состоит в следующем: в разделе 2 приведены основные определения и известные теоремы; в разделе 3 при любых значениях параметров для модели Поттса найдены инвариантные множества, в которых лежат все трансляционно-инвариантные меры Гиббса; в разделе 4 при некоторых условиях на параметры найдены аналогичные множества для модели SOS.

\section{2. ОПРЕДЕЛЕНИЯ И ИЗВЕСТНЫЕ ФАКТЫ}

Дерево Кэли $\mathfrak{I}^{k}$ порядка $k \geqslant 1$ - это бесконечное дерево, т. е. граф без циклов, из каждой вершины которого выходит ровно $k+1$ ребер. Пусть $\mathfrak{I}^{k}=(V, L, r)$, где $V$ - множество вершин графа $\mathfrak{I}^{k}, L$ - множество его ребер и $r$ - функция инцидентности, сопоставляющая каждому ребру $s \in L$ его концевые точки $x, y \in V$. Если $r(s)=\{x, y\}$, то вершины $x$ и $y$ называются ближайшими соседями; это обозначается как $s=\langle x, y\rangle$. Расстояние $d(x, y)$ между вершинами $x, y \in V$ на дереве Кэли определяется как минимальное значение $d$ такое, что найдутся вершины $x=x_{0}, x_{1}, \ldots, x_{d-1}, x_{d}=y$, последовательно являющиеся ближайшими соседями, т. е. $\left\langle x_{0}, x_{1}\right\rangle, \ldots,\left\langle x_{d-1}, x_{d}\right\rangle$ - ребра дерева Кэли.

Для фиксированного $x^{0} \in V$ положим

$$
\begin{gathered}
W_{n}=\left\{x \in V \mid d\left(x, x^{0}\right)=n\right\}, \quad V_{n}=\left\{x \in V \mid d\left(x, x^{0}\right) \leqslant n\right\}, \\
L_{n}=\left\{s=\langle x, y\rangle \in L \mid x, y \in V_{n}\right\} .
\end{gathered}
$$

Известно, что существует взаимно однозначное соответствие между множеством $V$ вершин дерева Кэли порядка $k \geqslant 1$ и группой $G_{k}$, являющейся свободным произведением $k+1$ циклических групп второго порядка с образующими $a_{1}, a_{2}, \ldots, a_{k+1}$.

Мы рассмотрим модель, в которой спиновые переменные принимают значения из множества $\Phi=\{1, \ldots, q\}, q \geqslant 2$, и расположены в вершинах дерева. Тогда конфигурация $\sigma$ на $V$ определяется как функция $x \in V \mapsto \sigma(x) \in \Phi$; множество всех конфигураций совпадает с $\Omega=\Phi^{V}$.

Гамильтониан модели Поттса определяется как

$$
H(\sigma)=-J \sum_{\langle x, y\rangle \in L} \delta_{\sigma(x) \sigma(y)},
$$

где $J \in \mathbb{R},\langle x, y\rangle$ - ближайшие соседи, а $\delta_{i j}$ - символ Кронекера.

Определим конечномерное распределение вероятностной меры $\mu$ в обьеме $V_{n}$ как

$$
\mu_{n}\left(\sigma_{n}\right)=Z_{n}^{-1} \exp \left\{-\beta H_{n}\left(\sigma_{n}\right)+\sum_{x \in W_{n}} h_{\sigma(x), x}\right\} \text {, }
$$


где $\beta=1 / T, T>0$ - температура, $Z_{n}^{-1}$ - нормировочный множитель,

$$
H_{n}\left(\sigma_{n}\right)=-J \sum_{\langle x, y\rangle \in L_{n}} \delta_{\sigma(x) \sigma(y)}
$$

и

$$
\left\{h_{x}=\left(h_{1, x}, \ldots, h_{q, x}\right) \in \mathbb{R}^{q}, x \in V\right\} .
$$

- совокупность векторов. Говорят, что вероятностное распределение (2) согласованное, если для всех $n \geqslant 1$ и $\sigma_{n-1} \in \Phi^{V_{n-1}}$

$$
\sum_{\omega_{n} \in \Phi^{W_{n}}} \mu_{n}\left(\sigma_{n-1} \vee \omega_{n}\right)=\mu_{n-1}\left(\sigma_{n-1}\right) .
$$

Здесь $\sigma_{n-1} \vee \omega_{n}$ есть объединение конфигураций. В этом случае существует единственная мера $\mu$ на $\Phi^{V}$ такая, что для всех $n$ и $\sigma_{n} \in \Phi^{V_{n}}$

$$
\mu\left(\left\{\left.\sigma\right|_{V_{n}}=\sigma_{n}\right\}\right)=\mu_{n}\left(\sigma_{n}\right) .
$$

Такая мера называется расщепленной мерой Гиббса, соответствующей гамильтониану (1) и векторнозначной функции $h_{x}, x \in V$.

В следующем утверждении [7] дается условие на функцию $h_{x}$, обеспечивающее согласованность меры $\mu_{n}\left(\sigma_{n}\right)$.

Теорема 1. Вероятностное распределение $\mu_{n}\left(\sigma_{n}\right), n=1,2, \ldots$, заданное формулой (2), является согласованным тогда и только тогда, когда для любого $x \in V$ имеет место следующее равенство:

$$
h_{x}=\sum_{y \in S(x)} F\left(h_{y}, \theta\right),
$$

где $\theta=e^{J \beta}, S(x)$ - множество прямых потомков точки $x$,

$$
S(x)=\left\{y \in W_{n+1} \mid d(x, y)=1\right\},
$$

бункиия

$$
F: h=\left(h_{1}, \ldots, h_{q-1}\right) \in \mathbb{R}^{q-1} \mapsto F(h, \theta)=\left(F_{1}, \ldots, F_{q-1}\right) \in \mathbb{R}^{q-1}
$$

задается формулами

$$
F_{i}=\ln \left(\frac{(\theta-1) e^{h_{i}}+\sum_{j=1}^{q-1} e^{h_{j}}+1}{\theta+\sum_{j=1}^{q-1} e^{h_{j}}}\right), \quad i=1, \ldots, q-1 .
$$

Пусть $\widehat{G}_{k}-$ подгруппа группы $G_{k}$.

ОПрЕДЕЛЕНиЕ 1. Совокупность векторов $h=\left\{h_{x}, x \in G_{k}\right\}$ называется $\widehat{G}_{k}$-периодической, если $h_{y x}=h_{x}$ для всех $x \in G_{k}, y \in \widehat{G}_{k} . G_{k}$-периодические совокупности называются трансляиионно-инвариантными.

ОпрЕдЕлЕниЕ 2. Мера $\mu$ называется $\widehat{G}_{k}$-периодической, если она соответствует $\widehat{G}_{k}$-периодической совокупности векторов $h$. 
Следующая теорема доказана в работе [15].

Теорема 2. Пусть $K$ - нормальный делитель конечного индекса в группе $G_{k}$. Тогда для модели Поттса все K-периодические меры Гиббса являются либо $G_{k}^{(2)}$ периодическими, либо транслячионно-инвариантными.

Помимо модели Поттса, мы рассмотрим модель SOS, в которой спиновые переменные принимают значения из множества $\Phi=\{0,1,2, \ldots, m\}, m \geqslant 2$, и расположены в вершинах дерева, а гамильтониан определяется как

$$
H(\sigma)=-J \sum_{\langle x, y\rangle \in L}|\sigma(x)-\sigma(y)|,
$$

где $J \in \mathbb{R},\langle x, y\rangle$ - ближайшие соседи, $\sigma(x) \in \Phi$ для любых $x \in V$.

Пусть $h: x \mapsto h_{x}=\left(h_{0, x}, h_{1, x}, \ldots, h_{m, x}\right) \in \mathbb{R}^{m+1}$ - векторнозначная функция переменной $x \in V \backslash\left\{x^{0}\right\}$. В следующем утверждении [14] дается условие на функцию $h_{x}$, обеспечивающее согласованность меры $\mu_{n}\left(\sigma_{n}\right)$.

ТЕОРема 3. Вероятностное распределение $\mu_{n}\left(\sigma_{n}\right), n=1,2, \ldots$, заданное формулой (2), является согласованным тогда и только тогда, когда для любой вериинъ $x \neq x^{0}$ имеет место равенство

$$
h_{x}^{*}=\sum_{y \in S(x)} F\left(h_{y}^{*}, m, \theta\right),
$$

где $\theta=e^{J \beta}, h_{x}^{*}=\left(h_{0, x}-h_{m, x}, h_{1, x}-h_{m, x}, \ldots, h_{m-1, x}-h_{m, x}\right)$ и бункция

$$
F(\cdot, m, \theta): \mathbb{R}^{m} \mapsto \mathbb{R}^{m}, \quad F(h, \theta, m)=\left(F_{0}(h, m, \theta), F_{1}(h, m, \theta), \ldots, F_{m-1}(h, m, \theta)\right),
$$

определяется формулами

$$
\begin{gathered}
F_{i}(h, m, \theta)=\ln \left(\frac{\sum_{j=0}^{m-1} \theta^{|i-j|} e^{h_{j}}+\theta^{m-i}}{\sum_{j=0}^{m-1} \theta^{m-j} e^{h_{j}}+1}\right), \quad i=0,1, \ldots, m-1, \\
h=\left(h_{0}, h_{1}, \ldots, h_{m-1}\right) .
\end{gathered}
$$

Заметим, что аналог теоремы 2 также справедлив для модели SOS (см. монографию [4], глава 6).

\section{3. ТРАНСЛЯЦИОННО-ИНВАРИАНТНЫЕ МЕРЫ ГИББСА ДЛЯ МОДЕЛИ ПОТТСА}

Рассмотрим трансляционно-инвариантные меры Гиббса, т. е. предположим, что $h_{x}=h=\left(h_{1}, \ldots, h_{q-1}\right) \in \mathbb{R}^{q-1}$ для любого $x \in V$. Тогда из уравнения (3) получим $h=k F(h, \theta)$, т. е.

$$
h_{i}=k \ln \left(\frac{(\theta-1) e^{h_{i}}+\sum_{j=1}^{q-1} e^{h_{j}}+1}{\theta+\sum_{j=1}^{q-1} e^{h_{j}}}\right), \quad i=1, \ldots, q-1 .
$$

Вводя обозначение $z_{i}=e^{h_{i}}, i=1, \ldots, q-1$, из (5) имеем

$$
z_{i}=\left(\frac{(\theta-1) z_{i}+\sum_{j=1}^{q-1} z_{j}+1}{\theta+\sum_{j=1}^{q-1} z_{j}}\right)^{k}, \quad i=1, \ldots, q-1 .
$$


Пусть $I \subset\{1, \ldots, q-1\}$. Введем обозначение

$$
M_{I}=\left\{z=\left(z_{1}, \ldots, z_{q-1}\right) \in \mathbb{R}^{q-1} \mid z_{i}=1 \text { для } i \in I, \quad z_{j}=z_{k} \text { для } j, k \notin I\right\} .
$$

Справедлива следующая теорема.

Теорема 4. При любых $q$ и $k$ все решения системы уравнений (6) с необходимостъю принадлежат множеству $M_{I}$, где $I \subset\{1, \ldots, q-1\}$.

ДокАзАтЕЛьСтво. Легко заметить, что $z_{i}=1, i=1, \ldots, q-1$, является решением системы (6).

Пусть $z_{i}=1$ для всех $i \in I$. Не нарушая общности, можно предполагать, что $I=\varnothing$. Положим $\sqrt[k]{z_{i}}=x_{i}, i=1, \ldots, q-1$. Тогда уравнения (6) запишутся следующим образом:

$$
x_{i}=\frac{(\theta-1) x_{i}^{k}+\sum_{j=1}^{q-1} x_{j}^{k}+1}{\sum_{j=1}^{q-1} x_{j}^{k}+\theta}, \quad i=1, \ldots, q-1 .
$$

Пусть $x_{i} \neq 1$ для всех $i=1, \ldots, q-1$. Из каждого уравнения (7) выразим $\theta$ и после некоторых преобразований получим

$$
\theta=\frac{\sum_{r=0}^{k} x_{i}^{r}+\sum_{j=1}^{q-1} x_{j}^{k}-x_{i}^{k}}{\sum_{r=1}^{k-1} x_{i}^{r}}, \quad i=1, \ldots, q-1
$$

Докажем, что $x_{i}=x_{i+1}$ для любых $i=1, \ldots, q-2$. В самом деле, из $i$-го и $(i+1)$-го уравнений (8) имеем

$$
\left(\sum_{r=0}^{k} x_{i}^{r}+\sum_{j=1}^{q-1} x_{j}^{k}-x_{i}^{k}\right) \sum_{r=1}^{k-1} x_{i+1}^{r}=\left(\sum_{r=0}^{k} x_{i+1}^{r}+\sum_{j=1}^{q-1} x_{j}^{k}-x_{i+1}^{k}\right) \sum_{r=1}^{k-1} x_{i}^{r}
$$

или, эквивалентно,

$$
\begin{gathered}
\left(\sum_{r=0}^{k} x_{i}^{r}+\sum_{j=1}^{q-1} x_{j}^{k}-x_{i}^{k}\right) \sum_{r=1}^{k-1} x_{i+1}^{r}-\left(\sum_{r=0}^{k} x_{i+1}^{r}+\sum_{j=1}^{q-1} x_{j}^{k}-x_{i+1}^{k}\right) \sum_{r=1}^{k-1} x_{i+1}^{r}+ \\
+\left(\sum_{r=0}^{k} x_{i+1}^{r}+\sum_{j=1}^{q-1} x_{j}^{k}-x_{i+1}^{k}\right) \sum_{r=1}^{k-1} x_{i+1}^{r}-\left(\sum_{r=0}^{k} x_{i+1}^{r}+\sum_{j=1}^{q-1} x_{j}^{k}-x_{i+1}^{k}\right) \sum_{r=1}^{k-1} x_{i}^{r}= \\
=\left(x_{i}-x_{i+1}\right) \sum_{s=0}^{k-2} \sum_{r=0}^{s} x_{i}^{r} x_{i+1}^{s-r}\left(-1-\sum_{j=1}^{q-1} x_{j}^{k}\right)=0 .
\end{gathered}
$$

Отсюда получим, что $x_{i}=x_{i+1}$, так как $x_{i}>0$ для всех $i=1, \ldots, q-1$. Следовательно, все решения системы (6) принадлежат множествам $M_{I}$. Теорема доказана.

Из теорем 1, 2 вытекает

СлЕДСТВИЕ 1. Все трансляционно-инвариантные меры Гиббса модели Поттса соответствуют решениям системы (6), которые лежат в инвариантных множествах $M_{I}$. 


\section{4. ТРАНСЛЯЦИОННО-ИНВАРИАНТНЫЕ МЕРЫ ГИББСА ДЛЯ МОДЕЛИ SOS}

Рассмотрим трансляционно-инвариантные меры Гиббса. Положив $z_{i}=e^{h_{i}}$, из соотношения (4) получим

$$
z_{i}=\left(\frac{\sum_{j=0}^{m-1} \theta^{|i-j|} z_{j}+\theta^{m-i}}{\sum_{j=0}^{m-1} \theta^{m-j} z_{j}+1}\right)^{k}, \quad i=0,1, \ldots, m-1 .
$$

Справедлива следующая

Теорема 5. Для модели SOS при $\theta>1 u k \geqslant 2$ справедливо следующее утверждение. При $m=2,3,4,5,6$ все решения системы уравнений (9) с необходимостью принадлежат множествам

$$
\begin{aligned}
& M_{1}=\left\{z=\left(z_{0}, z_{1}\right) \in \mathbb{R}^{2}: z_{0}=1,0<z_{1}<1\right\}, \\
& M_{2}=\left\{z=\left(z_{0}, z_{1}, z_{2}\right) \in \mathbb{R}^{3}: z_{0}=1, z_{1}=z_{2}\right\}, \\
& M_{3}=\left\{z=\left(z_{0}, z_{1}, z_{2}, z_{3}\right) \in \mathbb{R}^{4}: z_{0}=1, z_{1}=z_{3}\right\}, \\
& M_{4}=\left\{z=\left(z_{0}, z_{1}, z_{2}, z_{3}, z_{4}\right) \in \mathbb{R}^{5}: z_{0}=1, z_{1}=z_{4}, z_{2}=z_{3}\right\}, \\
& M_{5}=\left\{z=\left(z_{0}, z_{1}, z_{2}, z_{3}, z_{4}, z_{5}\right) \in \mathbb{R}^{6}: z_{0}=1, z_{1}=z_{5}, z_{2}=z_{4}\right\}
\end{aligned}
$$

соответственно.

ДокАЗАтЕЛЬство. Рассмотрим каждое из значений $m=2,3,4,5,6$ в отдельности.

1. Случай $m=2, k \geqslant 2$. Из уравнений (9) имеем

$$
z_{0}=\left(\frac{z_{0}+\theta z_{1}+\theta^{2}}{\theta^{2} z_{0}+\theta z_{1}+1}\right)^{k}, \quad z_{1}=\left(\frac{\theta z_{0}+z_{1}+\theta}{\theta^{2} z_{0}+\theta z_{1}+1}\right)^{k} .
$$

Введя обозначения $\sqrt[k]{z_{0}}=x, \sqrt[k]{z_{1}}=y$, после некоторых преобразований из (10) получим следующую систему уравнений:

$$
\begin{array}{r}
(x-1)\left(\left(\theta^{2}-1\right) \sum_{r=1}^{k-1} x^{r}+\theta^{2} x^{k}+\theta y^{k}+1\right)=0, \\
\theta^{2} x^{k} y+\theta\left(y^{k+1}-x^{k}-1\right)+y-y^{k}=0 .
\end{array}
$$

Из первого уравнения этой системы имеем $x=1$, следовательно, $z_{0}=1$. Тогда из второго уравнения системы (11) получим квадратное уравнение относительно $\theta$ :

$$
\theta^{2} y+\theta\left(y^{k+1}-2\right)+y-y^{k}=0
$$

причем его дискриминант

$$
D(y)=\left(y^{k+1}-2\right)^{2}-4 y\left(y-y^{k}\right)=y^{2 k+2}-4 y^{2}+4
$$

положителен. Действительно, $D(0)=4>0$. Найдем точку минимума дискриминанта. Имеем $D^{\prime}(y)=2 y\left[(k+1) y^{2 k}-4\right]=0$, отсюда

$$
y_{\min }=\sqrt[2 k]{\frac{4}{k+1}}, \quad D\left(y_{\min }\right)=\left(\frac{4}{k+1}\right)^{(k+1) / k}-\left(\frac{4}{k+1}\right)^{1 / k}+4>0
$$

при любом $k \geqslant 2$. 
Далее, рассмотрим решения уравнения (12):

$$
\theta_{1,2}=\frac{2-y^{k+1} \pm \sqrt{y^{2 k+2}-4 y^{2}+4}}{2 y}>1 .
$$

Следовательно, $y^{k+1}+2 y-2< \pm \sqrt{y^{2 k+2}-4 y^{2}+4}$.

Рассмотрим два случая. Пусть

$$
y^{k+1}+2 y-2<\sqrt{y^{2 k+2}-4 y^{2}+4} .
$$

Тогда, поскольку функция $\alpha(y)=y^{k+1}+2 y-2$ возрастает при $y>0$ и $\alpha(0)=-2$, $\alpha(1)=1, \alpha^{\prime \prime}(y)>0$, при $y^{k+1}+2 y-2 \leqslant 0$ получим решение $0<y \leqslant y^{*}<1$, где $y^{*}-$ корень уравнения $\alpha(y)=0$. Если же $y^{k+1}+2 y-2>0$, то, возведя обе части неравенства (13) в квадрат, получим систему неравенств

$$
y^{k+1}+2 y-2>0, \quad(y-1)\left(4 y^{k+1}+8 y\right)<0 .
$$

Ее решением является интервал $\left(y^{*}, 1\right)$, т. е. при выполнении условия (13) мы получим в качестве решения интервал $(0,1)$.

Пусть теперь

$$
y^{k+1}+2 y-2<-\sqrt{y^{2 k+2}-4 y^{2}+4} .
$$

Тогда, возведя в квадрат это неравенство при $y^{k+1}+2 y-2<0$, будем иметь систему неравенств

$$
y^{k+1}+2 y-2<0, \quad(y-1)\left(4 y^{k+1}+8 y\right)>0,
$$

которая не имеет решения, так как первое неравенство этой системы выполнено при $y \in\left(0, y^{*}\right)$, где $y^{*}<1$, а второе - при $y>1$.

Таким образом, мы имеем $z_{1} \in(0,1)$. При условии $z_{1} \in(0,1)$ верно и второе уравнение системы (10):

$$
z_{1}=\left(\frac{\theta z_{0}+z_{1}+\theta}{\theta^{2} z_{0}+\theta z_{1}+1}\right)^{k}<1 \quad \Longleftrightarrow \quad(\theta-1)\left(\theta z_{0}+z_{1}-1\right)>0 .
$$

Последнее неравенство верно при $z_{0}=1, \theta>1$.

Итак, система уравнений (10) имеет решение вида $z_{0}=1, z_{1} \in(0,1)$, следовательно, в случае $m=2, k \geqslant 2$ все решения системы (9) лежат в инвариантном множестве $M_{1}$.

2. Случай $m=3, k \geqslant 2$. Система уравнений (9) после введения обозначений $\sqrt[k]{z_{0}}=x_{0}, \sqrt[k]{z_{1}}=x_{1}, \sqrt[k]{z_{2}}=x_{2}$ принимает вид

$$
\begin{aligned}
& x_{0}\left(\theta^{3} x_{0}^{k}+\theta^{2} x_{1}^{k}+\theta x_{2}^{k}+1\right)=x_{0}^{k}+\theta x_{1}^{k}+\theta^{2} x_{2}^{k}+\theta^{3}, \\
& x_{1}\left(\theta^{3} x_{0}^{k}+\theta^{2} x_{1}^{k}+\theta x_{2}^{k}+1\right)=\theta x_{0}^{k}+x_{1}^{k}+\theta x_{2}^{k}+\theta^{2}, \\
& x_{2}\left(\theta^{3} x_{0}^{k}+\theta^{2} x_{1}^{k}+\theta x_{2}^{k}+1\right)=\theta^{2} x_{0}^{k}+\theta x_{1}^{k}+x_{2}^{k}+\theta .
\end{aligned}
$$


В первом уравнении системы (15) правую часть перенесем в левую и после некоторых преобразований получим

$$
\begin{aligned}
\theta^{3}\left(x_{0}^{k+1}-1\right)+x_{0}-x_{0}^{k}+\theta^{2} x_{0} x_{1}^{k}+\theta x_{0} x_{2}^{k}-\theta x_{1}^{k}-\theta^{2} x_{2}^{k}= \\
=\left[\theta^{3} \sum_{r=0}^{k} x_{0}^{r}-\sum_{r=0}^{k-1} x_{0}^{r}+1\right]\left(x_{0}-1\right)+ \\
\quad+\theta^{2}\left(x_{0} x_{1}^{k}-x_{1}^{k}+x_{1}^{k}-x_{2}^{k}\right)+\theta\left(x_{0} x_{2}^{k}-x_{2}^{k}+x_{2}^{k}-x_{1}^{k}\right)= \\
=\left[\left(\theta^{3}-1\right) \sum_{r=0}^{k-1} x_{0}^{r}+\theta^{3} x_{0}^{k}+\theta^{2} x_{1}^{k}+\theta x_{2}^{k}+1\right]\left(x_{0}-1\right)+ \\
\quad+\left(\theta^{2}-\theta\right) \sum_{s=0}^{k-1} x_{1}^{k-1-s} x_{2}^{s}\left(x_{1}-x_{2}\right)=0 .
\end{aligned}
$$

Далее, из второго уравнения системы (15) вычтем третье:

$$
\left(x_{1}-x_{2}\right)\left(\theta^{3} x_{0}^{k}+\theta^{2} x_{1}^{k}+\theta x_{2}^{k}+1\right)=\left(x_{0}^{k}-1\right)\left(\theta-\theta^{2}\right)+(1-\theta)\left(x_{1}^{k}-x_{2}^{k}\right)
$$

или, эквивалентно,

$$
\left(\theta^{2}-\theta\right) \sum_{r=0}^{k-1} x_{0}^{r}\left(x_{0}-1\right)+\left[\theta^{3} x_{0}^{k}+\theta^{2} x_{1}^{k}+\theta x_{2}^{k}+1+(\theta-1) \sum_{s=0}^{k-1} x_{1}^{k-1-s} x_{2}^{s}\right]\left(x_{1}-x_{2}\right)=0 .
$$

В итоге получим однородную систему уравнений относительно $x_{1}-x_{2}$ и $x_{0}-1$ :

$$
\begin{gathered}
{\left[L+\left(\theta^{3}-1\right) \sum_{r=0}^{k-1} x_{0}^{r}\right]\left(x_{0}-1\right)+\left(\theta^{2}-\theta\right) \sum_{s=0}^{k-1} x_{1}^{k-1-s} x_{2}^{s}\left(x_{1}-x_{2}\right)=0} \\
\left(\theta^{2}-\theta\right) \sum_{r=0}^{k-1} x_{0}^{r}\left(x_{0}-1\right)+\left[L+(\theta-1) \sum_{s=0}^{k-1} x_{1}^{k-1-s} x_{2}^{s}\right]\left(x_{1}-x_{2}\right)=0 .
\end{gathered}
$$

Ее определитель

$$
\left[L+\left(\theta^{3}-1\right) \sum_{r=0}^{k-1} x_{0}^{r}\right]\left[L+(\theta-1) \sum_{s=0}^{k-1} x_{1}^{k-1-s} x_{2}^{s}\right]-\left(\theta^{2}-\theta\right)^{2} \sum_{s=0}^{k-1} x_{1}^{k-1-s} x_{2}^{s} \cdot \sum_{r=0}^{k-1} x_{0}^{r}
$$

отличен от нуля при $\theta>1$, так как

$$
(\theta-1)\left(\theta^{3}-1\right)-\left(\theta^{2}-\theta\right)^{2}=(\theta-1)^{2}(\theta+1)>0 .
$$

Здесь $L=\theta^{3} x_{0}^{k}+\theta^{2} x_{1}^{k}+\theta x_{2}^{k}+1$. Таким образом, в случае $m=3, k \geqslant 2$ все решения системы (9) лежат в множестве $M_{2}$.

3. Случай $m=4, k \geqslant 2$. Введя обозначения $\sqrt[k]{z_{j}}=x_{j}, j=0,1,2,3$, перепишем систему уравнений (9) в виде

$$
\begin{aligned}
& x_{0}\left(\theta^{4} x_{0}^{k}+\theta^{3} x_{1}^{k}+\theta^{2} x_{2}^{k}+\theta x_{3}^{k}+1\right)=x_{0}^{k}+\theta x_{1}^{k}+\theta^{2} x_{2}^{k}+\theta^{3} x_{3}^{k}+\theta^{4}, \\
& x_{1}\left(\theta^{4} x_{0}^{k}+\theta^{3} x_{1}^{k}+\theta^{2} x_{2}^{k}+\theta x_{3}^{k}+1\right)=\theta x_{0}^{k}+x_{1}^{k}+\theta x_{2}^{k}+\theta^{2} x_{3}^{k}+\theta^{3}, \\
& x_{2}\left(\theta^{4} x_{0}^{k}+\theta^{3} x_{1}^{k}+\theta^{2} x_{2}^{k}+\theta x_{3}^{k}+1\right)=\theta^{2} x_{0}^{k}+\theta x_{1}^{k}+x_{2}^{k}+\theta x_{3}^{k}+\theta^{2}, \\
& x_{3}\left(\theta^{4} x_{0}^{k}+\theta^{3} x_{1}^{k}+\theta^{2} x_{2}^{k}+\theta x_{3}^{k}+1\right)=\theta^{3} x_{0}^{k}+\theta^{2} x_{1}^{k}+\theta x_{2}^{k}+x_{3}^{k}+\theta .
\end{aligned}
$$


Преобразуем первое уравнение этой системы, как в предыдущем случае, а из второго уравнения вычтем четвертое. В результате получим однородную систему уравнений относительно $x_{0}-1$ и $x_{1}-x_{3}$ :

$$
\begin{aligned}
& {\left[L_{1}+\left(\theta^{4}-1\right) \sum_{r=0}^{k-1} x_{0}^{r}\right]\left(x_{0}-1\right)+\left(\theta^{3}-\theta\right) \sum_{s=0}^{k-1} x_{1}^{k-1-s} x_{3}^{s}\left(x_{1}-x_{3}\right)=0,} \\
& \left(\theta^{3}-\theta\right) \sum_{r=0}^{k-1} x_{0}^{r}\left(x_{0}-1\right)+\left[L_{1}+\left(\theta^{2}-1\right) \sum_{s=0}^{k-1} x_{1}^{k-1-s} x_{3}^{s}\right]\left(x_{1}-x_{3}\right)=0 .
\end{aligned}
$$

Ее определитель

$$
\left|\begin{array}{cc}
L_{1}+\left(\theta^{4}-1\right) \sum_{r=0}^{k-1} x_{0}^{r} & \left(\theta^{3}-\theta\right) \sum_{s=0}^{k-1} x_{1}^{k-1-s} x_{3}^{s} \\
\left(\theta^{3}-\theta\right) \sum_{r=0}^{k-1} x_{0}^{r} & L_{1}+\left(\theta^{2}-1\right) \sum_{s=0}^{k-1} x_{1}^{k-1-s} x_{3}^{s}
\end{array}\right|
$$

отличен от нуля при $\theta>1$, а точнее, строго больше нуля, так как

$$
\left(\theta^{4}-1\right)\left(\theta^{2}-1\right)-\left(\theta^{3}-\theta\right)^{2}=\left(\theta^{2}-1\right)^{2}>0 .
$$

Здесь $L_{1}=\theta^{4} x_{0}^{k}+\theta^{3} x_{1}^{k}+\theta^{2} x_{2}^{k}+\theta x_{3}^{k}+1$. Следовательно, в случае $m=4, k \geqslant 2$ система уравнений (9) имеет только решения вида $z_{0}=1, z_{1}=z_{3}$, т. е. все решения лежат в инвариантном множестве $M_{3}$.

4. Случай $m=5, k \geqslant 2$. Аналогично предыдущему случаю, введя обозначения $\sqrt[k]{z_{j}}=x_{j}, j=0,1,2,3,4$, перепишем систему уравнений (9) как

$$
\begin{aligned}
& x_{0}\left(\theta^{5} x_{0}^{k}+\theta^{4} x_{1}^{k}+\theta^{3} x_{2}^{k}+\theta^{2} x_{3}^{k}+\theta x_{4}^{k}+1\right)=x_{0}^{k}+\theta x_{1}^{k}+\theta^{2} x_{2}^{k}+\theta^{3} x_{3}^{k}+\theta^{4} x_{4}^{k}+\theta^{5}, \\
& x_{1}\left(\theta^{5} x_{0}^{k}+\theta^{4} x_{1}^{k}+\theta^{3} x_{2}^{k}+\theta^{2} x_{3}^{k}+\theta x_{4}^{k}+1\right)=\theta x_{0}^{k}+x_{1}^{k}+\theta x_{2}^{k}+\theta^{2} x_{3}^{k}+\theta^{3} x_{4}^{k}+\theta^{4}, \\
& x_{2}\left(\theta^{5} x_{0}^{k}+\theta^{4} x_{1}^{k}+\theta^{3} x_{2}^{k}+\theta^{2} x_{3}^{k}+\theta x_{4}^{k}+1\right)=\theta^{2} x_{0}^{k}+\theta x_{1}^{k}+x_{2}^{k}+\theta x_{3}^{k}+\theta^{2} x_{4}^{k}+\theta^{3}, \\
& x_{3}\left(\theta^{5} x_{0}^{k}+\theta^{4} x_{1}^{k}+\theta^{3} x_{2}^{k}+\theta^{2} x_{3}^{k}+\theta x_{4}^{k}+1\right)=\theta^{3} x_{0}^{k}+\theta^{2} x_{1}^{k}+\theta x_{2}^{k}+x_{3}^{k}+\theta x_{4}^{k}+\theta^{2}, \\
& x_{4}\left(\theta^{5} x_{0}^{k}+\theta^{4} x_{1}^{k}+\theta^{3} x_{2}^{k}+\theta^{2} x_{3}^{k}+\theta x_{4}^{k}+1\right)=\theta^{4} x_{0}^{k}+\theta^{3} x_{1}^{k}+\theta^{2} x_{2}^{k}+\theta x_{3}^{k}+x_{4}^{k}+\theta .
\end{aligned}
$$

В первом уравнении этой системы правую часть перенесем в левую и сделаем некоторые преобразования, получим

$$
\begin{aligned}
\theta^{5}\left(x_{0}^{k+1}-1\right)+x_{0}-x_{0}^{k}+\theta^{4}\left(x_{0} x_{1}^{k}-x_{4}^{k}\right)+ \\
\quad+\theta^{3}\left(x_{0} x_{2}^{k}-x_{3}^{k}\right)+\theta^{2}\left(x_{0} x_{3}^{k}-x_{2}^{k}\right)+\theta\left(x_{0} x_{4}^{k}-x_{1}^{k}\right)= \\
=\left[\theta^{5} \sum_{r=0}^{k} x_{0}^{r}-x_{0} \sum_{r=0}^{k-2} x_{0}^{r}\right]\left(x_{0}-1\right)+\theta^{4}\left(x_{0} x_{1}^{k}-x_{1}^{k}+x_{1}^{k}-x_{4}^{k}\right)+ \\
\quad+\theta^{3}\left(x_{0} x_{2}^{k}-x_{2}^{k}+x_{2}^{k}-x_{3}^{k}\right)+\theta^{2}\left(x_{0} x_{3}^{k}-x_{3}^{k}+x_{3}^{k}-x_{2}^{k}\right)+\theta\left(x_{0} x_{4}^{k}-x_{4}^{k}+x_{4}^{k}-x_{1}^{k}\right)=
\end{aligned}
$$




$$
\begin{aligned}
= & {\left[\theta^{5} x_{0}^{k}+\left(\theta^{5}-1\right) \sum_{r=0}^{k-1} x_{0}^{r}+1\right]\left(x_{0}-1\right)+\theta^{4}\left[x_{1}^{k}\left(x_{0}-1\right)+\left(x_{1}-x_{4}\right) \sum_{s=0}^{k-1} x_{1}^{k-1-s} x_{4}^{s}\right]+} \\
& +\theta^{3}\left[x_{2}^{k}\left(x_{0}-1\right)+\left(x_{2}-x_{3}\right) \sum_{s=0}^{k-1} x_{2}^{k-1-s} x_{3}^{s}\right]+ \\
& +\theta^{2}\left[x_{3}^{k}\left(x_{0}-1\right)+\left(x_{3}-x_{2}\right) \sum_{s=0}^{k-1} x_{2}^{k-1-s} x_{3}^{s}\right]+ \\
& +\theta\left[x_{4}^{k}\left(x_{0}-1\right)+\left(x_{4}-x_{1}\right) \sum_{s=0}^{k-1} x_{1}^{k-1-s} x_{4}^{s}\right]=0
\end{aligned}
$$

или, эквивалентно,

$$
\begin{aligned}
& {\left[L_{2}+\left(\theta^{5}-1\right) \sum_{r=0}^{k-1} x_{0}^{r}\right]\left(x_{0}-1\right)+\left(\theta^{4}-\theta\right) \sum_{s=0}^{k-1} x_{1}^{k-1-s} x_{4}^{s}\left(x_{1}-x_{4}\right)+} \\
& \quad+\left(\theta^{3}-\theta^{2}\right) \sum_{s=0}^{k-1} x_{2}^{k-1-s} x_{3}^{s}\left(x_{2}-x_{3}\right)=0
\end{aligned}
$$

где $L_{2}=\theta^{5} x_{0}^{k}+\theta^{4} x_{1}^{k}+\theta^{3} x_{2}^{k}+\theta^{2} x_{3}^{k}+\theta x_{4}^{k}+1$. Далее, из второго уравнения системы (17) вычтем пятое, из третьего - четвертое и в результате получим однородную систему уравнений относительно $x_{0}-1, x_{1}-x_{4}$ и $x_{2}-x_{3}$ :

$$
\begin{aligned}
& {\left[L_{2}+\left(\theta^{5}-1\right) \sum_{r=0}^{k-1} x_{0}^{r}\right]\left(x_{0}-1\right)+\left(\theta^{4}-\theta\right) \sum_{s=0}^{k-1} x_{1}^{k-1-s} x_{4}^{s}\left(x_{1}-x_{4}\right)+} \\
& \quad+\left(\theta^{3}-\theta^{2}\right) \sum_{s=0}^{k-1} x_{2}^{k-1-s} x_{3}^{s}\left(x_{2}-x_{3}\right)=0 \\
& \left(\theta^{4}-\theta\right) \sum_{r=0}^{k-1} x_{0}^{r}\left(x_{0}-1\right)+\left[L_{2}+\left(\theta^{3}-1\right) \sum_{s=0}^{k-1} x_{1}^{k-1-s} x_{4}^{s}\right]\left(x_{1}-x_{4}\right)+ \\
& \quad+\left(\theta^{2}-\theta\right) \sum_{s=0}^{k-1} x_{2}^{k-1-s} x_{3}^{s}\left(x_{2}-x_{3}\right)=0 \\
& \left(\theta^{3}-\theta^{2}\right) \sum_{r=0}^{k-1} x_{0}^{r}\left(x_{0}-1\right)+\left(\theta^{2}-\theta\right) \sum_{s=0}^{k-1} x_{1}^{k-1-s} x_{4}^{s}\left(x_{1}-x_{4}\right)+ \\
& \quad+\left[L_{2}+(\theta-1) \sum_{s=0}^{k-1} x_{2}^{k-1-s} x_{3}^{s}\right]\left(x_{2}-x_{3}\right)=0 .
\end{aligned}
$$

Легко проверить, что определитель этой системы

$$
\left|\begin{array}{ccc}
L_{2}+\left(\theta^{5}-1\right) \sum_{r=0}^{k-1} x_{0}^{r} & \left(\theta^{4}-\theta\right) \sum_{s=0}^{k-1} x_{1}^{k-1-s} x_{4}^{s} & \left(\theta^{3}-\theta^{2}\right) \sum_{s=0}^{k-1} x_{2}^{k-1-s} x_{3}^{s} \\
\left(\theta^{4}-\theta\right) \sum_{r=0}^{k-1} x_{0}^{r} & L_{2}+\left(\theta^{3}-1\right) \sum_{s=0}^{k-1} x_{1}^{k-1-s} x_{4}^{s} & \left(\theta^{2}-\theta\right) \sum_{r=0}^{k-1} x_{2}^{k-1-s} x_{3}^{s} \\
\left(\theta^{3}-\theta^{2}\right) \sum_{r=0}^{k-1} x_{0}^{r} & \left(\theta^{2}-\theta\right) \sum_{r=0}^{k-1} x_{1}^{k-1-s} x_{4}^{s} & L_{2}+(\theta-1) \sum_{s=0}^{k-1} x_{2}^{k-1-s} x_{3}^{s}
\end{array}\right|
$$

2 Теоретическая и математическая физика, т. 179, № 1, 2014 г. 
отличен от нуля при $\theta>1$, так как коэффициент при $L_{2} \sum_{r=0}^{k-1} x_{0}^{r} \cdot \sum_{s=0}^{k-1} x_{2}^{k-1-s} x_{3}^{s}$ равен

$$
\left(\theta^{5}-1\right)(\theta-1)-\left(\theta^{3}-\theta^{2}\right)^{2}=(\theta-1)^{2}\left(\theta^{3}+\theta^{2}+\theta+1\right)>0,
$$

коэффициент при $L_{2} \sum_{r=0}^{k-1} x_{0}^{r} \cdot \sum_{s=0}^{k-1} x_{1}^{k-1-s} x_{4}^{s}$ равен

$$
\left(\theta^{3}-1\right)\left(\theta^{5}-1\right)-\left(\theta^{4}-\theta\right)^{2}=(\theta-1)^{2}\left(\theta^{2}+\theta+1\right)\left(\theta^{4}+\theta^{3}+\theta+1\right)>0,
$$

коэффициент при $L_{2} \sum_{s=0}^{k-1} x_{1}^{k-1-s} x_{4}^{s} \cdot \sum_{l=0}^{k-1} x_{2}^{k-1-l} x_{3}^{l}$ равен

$$
\left(\theta^{3}-1\right)(\theta-1)-\left(\theta^{2}-\theta\right)^{2}=(\theta-1)^{2}>0,
$$

и наконец, коэффициент при $\sum_{r=0}^{k-1} x_{0}^{r} \cdot \sum_{s=0}^{k-1} x_{1}^{k-1-s} x_{4}^{s} \cdot \sum_{l=0}^{k-1} x_{2}^{k-1-l} x_{3}^{l}$ равен

$$
\begin{gathered}
(\theta-1)\left(\theta^{3}-1\right)\left(\theta^{5}-1\right)+2\left(\theta^{2}-\theta\right)\left(\theta^{3}-\theta^{2}\right)\left(\theta^{4}-\theta\right)-\left(\theta^{3}-\theta\right)\left(\theta^{3}-\theta^{2}\right)^{2}- \\
-(\theta-1)\left(\theta^{4}-\theta\right)^{2}-\left(\theta^{5}-1\right)\left(\theta^{2}-\theta\right)^{2}=(\theta-1)^{3}\left(\theta^{4}+\theta^{2}+2 \theta+1\right)>0,
\end{gathered}
$$

а остальные слагаемые положительны. Итак, в случае $m=5, k \geqslant 2$ система уравнений (9) имеет только решения вида $z_{0}=1, z_{1}=z_{4}, z_{2}=z_{3}$.

5 . Случай $m=6, k \geqslant 2$. Введя обозначения $\sqrt[k]{z_{j}}=x_{j}, j=0,1,2,3,4,5$, перепишем систему уравнений (9) как

$$
\begin{aligned}
& x_{0}\left(\theta^{6} x_{0}^{k}+\theta^{5} x_{1}^{k}+\cdots+\theta x_{5}^{k}+1\right)=x_{0}^{k}+\theta x_{1}^{k}+\theta^{2} x_{2}^{k}+\theta^{3} x_{3}^{k}+\theta^{4} x_{4}^{k}+\theta^{5} x_{5}^{k}+\theta^{6}, \\
& x_{1}\left(\theta^{6} x_{0}^{k}+\theta^{5} x_{1}^{k}+\cdots+\theta x_{5}^{k}+1\right)=\theta x_{0}^{k}+x_{1}^{k}+\theta x_{2}^{k}+\theta^{2} x_{3}^{k}+\theta^{3} x_{4}^{k}+\theta^{4} x_{5}^{k}+\theta^{5}, \\
& x_{2}\left(\theta^{6} x_{0}^{k}+\theta^{5} x_{1}^{k}+\cdots+\theta x_{5}^{k}+1\right)=\theta^{2} x_{0}^{k}+\theta x_{1}^{k}+x_{2}^{k}+\theta x_{3}^{k}+\theta^{2} x_{4}^{k}+\theta^{3} x_{5}^{k}+\theta^{4}, \\
& x_{3}\left(\theta^{6} x_{0}^{k}+\theta^{5} x_{1}^{k}+\cdots+\theta x_{5}^{k}+1\right)=\theta^{3} x_{0}^{k}+\theta^{2} x_{1}^{k}+\theta x_{2}^{k}+x_{3}^{k}+\theta x_{4}^{k}+\theta 2 x_{5}^{k}+\theta^{3}, \\
& x_{4}\left(\theta^{6} x_{0}^{k}+\theta^{5} x_{1}^{k}+\cdots+\theta x_{5}^{k}+1\right)=\theta^{4} x_{0}^{k}+\theta^{3} x_{1}^{k}+\theta^{2} x_{2}^{k}+\theta x_{3}^{k}+x_{4}^{k}+\theta x_{5}^{k}+\theta^{2}, \\
& x_{5}\left(\theta^{6} x_{0}^{k}+\theta^{5} x_{1}^{k}+\cdots+\theta x_{5}^{k}+1\right)=\theta^{5} x_{0}^{k}+\theta^{4} x_{1}^{k}+\theta^{3} x_{2}^{k}+\theta^{2} x_{3}^{k}+\theta x_{4}^{k}+x_{5}^{k}+\theta .
\end{aligned}
$$

Аналогично предыдущим случаям в этой системе уравнений преобразуем первое уравнение и вычтем из второго уравнения шестое, а из третьего - пятое. В результате получим однородную систему уравнений относительно $x_{0}-1, x_{1}-x_{5}$ и $x_{2}-x_{4}$, определитель которой имеет вид

$$
\left|\begin{array}{ccc}
L_{3}+\left(\theta^{6}-1\right) a & \left(\theta^{5}-\theta\right) b & \left(\theta^{4}-\theta^{2}\right) c \\
\left(\theta^{5}-\theta\right) a & L_{3}+\left(\theta^{4}-1\right) b & \left(\theta^{3}-\theta\right) c \\
\left(\theta^{4}-\theta^{2}\right) a & \left(\theta^{3}-\theta\right) b & L_{3}+\left(\theta^{2}-1\right) c
\end{array}\right|
$$

где

$$
a=\sum_{r=1}^{k-1} x_{0}^{r}, \quad b=\sum_{s=0}^{k-1} x_{1}^{k-1-s} x_{5}^{s}, \quad c=\sum_{l=0}^{k-1} x_{2}^{k-1-l} x_{4}^{l}
$$

и $L_{3}=\theta^{6} x_{0}^{k}+\theta^{5} x_{1}^{k}+\cdots+\theta x_{5}^{k}+1$. Легко проверить, что этот определитель больше нуля при $\theta>1$. Следовательно, в случае $m=6, k \geqslant 2$ система уравнений (9) имеет решения, которые лежат в инвариантном множестве $M_{5}$. Теорема доказана полностью.

СлеДСТвИЕ 2. При $m=2,3,4,5,6$ все трансляционно-инвариантные меры Гиббса модели SOS соответствуют решениям системы (9), которые лежат в инвариантных множествах $M_{i}, i=1,2,3,4,5$, соответственно. 
Благодарности. Автор благодарит профессора У.А. Розикова за постановку задачи и полезные советы.

\section{Список литературы}

[1] Х.-О. Георги, Гиббсовские меры и фазовые переходы, Мир, М., 1992.

[2] C. J. Preston, Gibbs States on Countable Sets, Cambridge Tracts in Mathematics, 68, Cambridge Univ. Press, Cambridge, 1974.

[3] Я. Г. Синай, Теория фазовых переходов. Строгие результаты, Наука, М., 1980.

[4] U. A. Rozikov, Gibbs Measures on Cayley Trees, World Sci., Singapore, 2013.

[5] Н. Н. Ганиходжаев, ТМФ, 85:2 (1990), 163-175.

[6] Н. Н. Ганиходжаев, ДАН РУз, 6-7 (1992), 4-7.

[7] Н. Н. Ганиходжаев, У. А. Розиков, ТМФ, 111:1 (1997), 109-117.

[8] N. N. Ganikhodjaev, U. A. Rozikov, Lett. Math. Phys., 75:2 (2006), 99-109.

[9] F. S. de Aguiar, F. A. Bosco, A. S. Martinez, R.S. Goulart, Jr., J. Statist. Phys., 58:5-6 (1990), 1231-1238.

[10] F. S. de Aguiar, L. B. Bernardes, R. S. Goulart, Jr., J. Statist. Phys., 64:3-4 (1991), 673-682.

[11] А. В. Бакаев, А. Н. Ермилов, А. М. Курбатов, Докл. АН СССР, 299:3 (1988), 603-606.

[12] F. Wagner, D. Grensing, J. Heide, J. Phys. A, 34:50 (2001), 11261-11272.

[13] A. E. Mazel, Yu. M. Suhov, J. Statist. Phys., 64:1-2 (1991), 111-134.

[14] U.A. Rozikov, Yu. M. Suhov, Infin. Dimens. Anal. Quantum Probab. Relat. Top., 9:3 (2006), 471-488.

[15] У. А. Розиков, Р. М. Хакимов, ТМФ, 175:2 (2013), 300-312.

Поступила в редакцию 2.10.2013 